\title{
Informed Choice of Contraceptive Methods among Women in Ethiopia: Further Analysis of the 2016 Ethiopian Demographic Health Survey
}

This article was published in the following Dove Press journal: Open Access Journal of Contraception

\author{
Simegnew Handebo (1D \\ Department of Health Education and \\ Behavioral Sciences, Institute of Public \\ Health, College of Medicine and Health \\ Sciences, University of Gondar, Gondar, \\ Ethiopia
}

Background: Delivering modern contraceptive methods only may not provide much insight into the quality of family-planning services. Informed choice emphasizes that clients select the method that best satisfies their needs by informing them about all available contraceptive methods, side effects of the methods, and how to deal with the side effects if experienced. This study investigated informed choice regarding contraceptives and associated factors among women in Ethiopia.

Methods: Data were extracted from the 2016 Ethiopian Demographic and Health Survey. Information on informed choice was extracted from the individual women's data. Subjects comprised 3,511 (weighted) reproductive-age women aged 15-49 years using selected contraceptive methods. Bivariate and multivariate logistic regression models were used to assess factors associated with informed of contraceptive choice among women in Ethiopia. $P<0.05$ was considered statistically significant.

Results: Only $36.2 \%$ of the participants were informed on contraceptive methods. Increased age, watching television less than once per week, and visiting health facilities in the last 12 months were associated with higher odds of being informed on contraceptive methods. Being affiliated with other religious groups, primary education only, having a husband employed in agriculture, being unskilled, with an unknown job, living in Amhara and Oromia regions, watching television at least once a week, and receiving the service from private clinics and pharmacies were associated with lower odds of being informed on contraceptive methods.

Conclusion: A substantial proportion of reproductive-age women in Ethiopia were inadequately informed about side effects or other methods. This varied by socio-demographic factors, sources of contraception, method, and frequency of visiting health institutions. This study suggests that interventions that increase family-planning counseling to the level that clients understand the methods are needed. Private health facilities also need to focus on delivering essential messages about methods they provide and assure women's rights and choices.

Keywords: informed choice, contraceptive methods, reproductive-age women, Ethiopia

\section{Background}

The 1994 International Conference on Population and Development in Cairo declared sexual and reproductive health a human right. Family planning (FP) is a core part of the broader context of reproductive health and rights. ${ }^{1,2}$ The London FP Summit of 2012 brought the global initiative for universal access to FP services to the world, focusing on voluntary service, quality of care, and meeting the unmet FP needs. ${ }^{3}$ The principle of informed choice has been a cornerstone of reproductive
Correspondence: Simegnew Handebo PO Box 196

Tel +25I-9I-249-0659

Email hsimegnew@yahoo.com

Open Access Journal of Contraception 2021:|2 83-9| 
rights and a fundamental tenet of quality FP service. ${ }^{4}$ Informed choice emphasizes that clients select the method that best satisfies their personal, reproductive, and health needs, based on a thorough understanding of contraceptive options. $^{5}$

FP information and services are an essential component of the health and rights of all individuals. ${ }^{6}$ Anyone seeking FP services has the right to be fully informed on the benefits, potential adverse effects, and available alternatives by trained personnel. ${ }^{6,7}$ The health system is responsible for providing proper and noncoercive counseling, accurate information, competent providers, and a range of methods to facilitate full, free, and informed decision-making about contraception. Censoring, withholding, or intentionally misrepresenting information about contraceptive methods can put health and human rights in jeopardy. ${ }^{8,9}$ Sates should also ensure that third parties do not limit people's access to health-related information and services. ${ }^{9}$

Since the quality of FP services is one of the aspects that determine continual use of a method, providers should understand the concept of sexual and reproductive rights and their role in helping clients exercise these rights. Contraception-service users need to understand that they have the right to ask questions, get answers, and make their own decisions. However, informed choice is constrained by lack of understanding of the concept of informed choice, as well as by social norms that limit women's and adolescents' ability to assert their rights. ${ }^{10-12}$

In Ethiopia, contraceptive methods are primarily distributed throughout the public sector free of charge. ${ }^{13} \mathrm{FP}$ is seen as a key policy objective, with targets included in many health and national strategies and plans. FP is a central component of the National Reproductive Health Strategy (2006-2015), Health Sector Transformation Plan $\mathrm{V}$, and Growth and Transformation Plan of the country. For instance, increasing FP services is one of eight targets set in the Growth and Transformation Plan for health. ${ }^{14}$

The quality of FP service is critical for women to make informed reproductive and sexual health decisions. Service providers play a vital role in enhancing women's informed contraceptive decision-making. ${ }^{10}$ In Ethiopia, quality of service is positively correlated with modern contraceptive use. ${ }^{13,15}$ According to the 2016 Ethiopian Demographic and Health Survey (EDHS), only 35\% of married women used modern contraceptives. More than a third of these (35\%) had discontinued use within 12 months. Evidence revealed side effects and health concerns were the major reasons for discontinuation. ${ }^{16,17}$ Service providers play an active role in enhancing use and reducing discontinuation via informing women about method-related side effects and health concerns. ${ }^{5,10}$

As such, doubts remain regarding the extent to which contraceptive methods are promoted by informed choice of women. Numerous studies have been done on the use and discontinuation of contraceptive methods in Ethiopia; however, the issue of informed choice, one of the important indicators of quality of service, has not been well investigated. Knowing the extent of informed choice of a method and associated factors is a key component in designing successful interventions to improve the quality of the service. Therefore, this study assessed the prevalence of informed choice of contraceptive methods and associated factors among reproductive-age women in Ethiopia.

\section{Methods}

\section{Data and Study Setting}

A secondary data analysis was conducted using the 2016 EDHS data. The EDHS holds data that is publicly available via Measure DHS at https://dhsprogram.com/data/ available-datasets.cfm. The data were collected by the Ethiopia Central Statistical Agency with technical assistance from the International Classification of Functioning, Disability, and Health, international sources, and funding through the US Agency for International Development. The DHS is a large-scale cross-sectional household survey that collects information from nationally representative samples. The survey follows an international methodological approach and is conducted every 5 years. It focuses on maternal and child health, reproductive health, fertility, nutrition, mortality, and adults' health-behavior indicators. This study was conducted from January 18, 2016 to June 27, 2016. ${ }^{16}$

\section{Sampling Procedure}

A two-stage stratified cluster-sampling technique was employed. In the first stage, a total of 645 enumeration areas (202 urban and 443 rural) were selected based on the 2007 population housing census. In the second stage, 28 households per enumeration area were selected using systematic random sampling, with equal-probability selection per enumeration area. From 16,583 eligible women identified for individual interviews, 15,683 completed the interview, yielding a response rate of $95 \%$. 
Detailed methodology is found elsewhere. ${ }^{16}$ Reproductive-age women who used selected contraceptive methods, ie, female sterilization, implants, injections, IUD, and the pill, were included. Nonusers and those who used methods other than the five just listed were excluded. Therefore, a total of 3,511 (weighted) women using the contraceptive methods selected were included in the analysis.

\section{Study Variables}

The outcome variable of this study was informed choice of contraceptive methods. Data on informed choices were collected among women who used one of the five modern methods (female sterilization, implants, injections, IUD, and the pill). It was constructed based on the responses provided to questions in the EDHS questionnaire. Description and measurement of the outcome variables used in the study are presented in Table 1.

Based on the literature, ${ }^{16-24}$ age, marital status, residence, administrative region, education, occupation, religion, husband's educatiol, husband's occupation, wealth, frequency of media use (radio, television, and newspapers), access to FP messages, sources of FP methods, and visiting health institutions in the past 12 months were selected explanatory variables for analysis.

\section{Data Processing and Analysis}

Data were cleaned and analyzed using Stata 14. Sample weight was applied for all analysis. Multicollinearity among independent variables was checked using varianceinflation factors. Descriptive statistics were used to describe the characteristics of study participants, and result are presented as text and tables. Initially, bivariate logistic regression analysis was performed, and variables with a $P \leq 0.2$ were included in the multivariate logistic regression model. AORs with $95 \%$ CIs were estimated to identify factors associated with informed choice for contraception in Ethiopia. $P<0.05$ was taken as statistically significant.

\section{Results}

A total of 3,511 reproductive-aged women were included in the analysis. Among these, 985 (28.05\%) were aged 2529 years. The mean age was $29.27 \pm 7.31$ years. All women were married. A majority $(2,685,76.5 \%)$ lived in rural areas, and 1,804 (51.4\%) and 1,592 (45.4\%) were uneducated and unemployed, respectively. About a third $(1,137$, 32.39) were from the Amhara region (Table 2).

Women's exposure to mass media was measured by frequency of watching television, listening to the radio, and reading the newspaper. A total of $626(17.8 \%)$ and 549 $(15.6 \%)$ listened to the radio and watched television at least once a week, respectively. Fewer (99, 2.8\%) read a newspaper at least once a week, while 942 (26.8\%) and 636 (18.1\%) heard about FP from radio and television, respectively. On the other hand, 1,563 (44.5\%) heard about FP from community events/conversations (Table 3 ).

Injectables $(2,247,64 \%)$ were the most common contraceptive method for women. Government health institutions $(2,922,83.4 \%)$ were the main sources of modern contraceptive methods in Ethiopia. A majority (2,369, $72.51 \%$ ) decided on methods with their husbands. Of current users, $1,599(45.5 \%)$ and 1,950 (55.6\%) were informed about potential side effects and other available FP methods, respectively. From those informed about side effects, a majority $(1,140,81 \%)$ had been told what to do when experiencing side effects. The overall prevalence of

Table I Description and Measurements of Outcome Variables Used in the Estimations

\begin{tabular}{|l|l|l|}
\hline Variable & \multicolumn{1}{|c|}{ Description } & \multicolumn{1}{|c|}{ Measurements } \\
\hline $\begin{array}{l}\text { Counseled about side } \\
\text { effect }\end{array}$ & $\begin{array}{l}\text { "When you started using the current method, were you told about side } \\
\text { effects or problems you might have with the method?" and "Were you ever } \\
\text { told by a health or family-planning worker about side effects or problems you } \\
\text { might have with the method?" }\end{array}$ & $\begin{array}{l}\text { "Yes" to either of question = I } \\
\text { (considered informed), "No" = 0. }\end{array}$ \\
\hline $\begin{array}{l}\text { Informed about other } \\
\text { available methods }\end{array}$ & $\begin{array}{l}\text { "When you started using the current method, were you told about other } \\
\text { methods of familyplanning that you could use?", and "Were you ever told by } \\
\text { a health or family-planning worker about other methods of family planning } \\
\text { that you could use?" }\end{array}$ & $\begin{array}{l}\text { "Yes" to either of these two questions = } \\
\text { (considered informed), "No" = 0. }\end{array}$ \\
\hline $\begin{array}{l}\text { Informed choice for } \\
\text { contraception } \\
\text { (outcome) }\end{array}$ & $\begin{array}{l}\text { Percentage of women who were informed about both side effects and other } \\
\text { available methods }\end{array}$ & Yes = I, no =0 \\
\hline
\end{tabular}


Table 2 Sociodemographic Characteristics of Reproductive Age Women Currently Using Selected Contraceptive Methods in Ethiopia, EDHS 2016

\begin{tabular}{|c|c|c|}
\hline & & Weighted Frequency (\%) \\
\hline \multirow[t]{7}{*}{ Age (years) } & $15-19$ & $245(6.99)$ \\
\hline & $20-24$ & $710(20.23)$ \\
\hline & $25-29$ & $985(28.05)$ \\
\hline & $30-34$ & 691 (19.69) \\
\hline & $35-39$ & $482(13.73)$ \\
\hline & $40-44$ & $286(8.16)$ \\
\hline & $45-49$ & III (3.16) \\
\hline \multirow[t]{2}{*}{ Residence } & Urban & $826(23.52)$ \\
\hline & Rural & $2,685(76.48)$ \\
\hline \multirow[t]{4}{*}{ Religion } & Orthodox & $\mathrm{I}, 838(52.34)$ \\
\hline & Muslim & 725 (20.64) \\
\hline & Protestant & 894 (25.48) \\
\hline & Other & $54(1.54)$ \\
\hline \multirow[t]{4}{*}{ Education } & None & $\mathrm{I}, 804$ (5I.39) \\
\hline & Primary & I,I 49 (32.7I) \\
\hline & Secondary & 333 (9.49) \\
\hline & Higher & $225(6.4 I)$ \\
\hline \multirow[t]{5}{*}{ Occupation } & Not working & I,592 (45.35) \\
\hline & Professional & I,0।4 (28.89) \\
\hline & Agriculture & $785(22.36)$ \\
\hline & Unskilled & $41(1.16)$ \\
\hline & Others & $79(2.24)$ \\
\hline \multirow{5}{*}{$\begin{array}{l}\text { Husband's } \\
\text { occupation }\end{array}$} & Not working & 157 (4.79) \\
\hline & Professional & 961 (29.42) \\
\hline & Agriculture & I,9I5 (58.6I) \\
\hline & Unskilled & 108 (3.32) \\
\hline & Others & $126(3.87)$ \\
\hline \multirow{5}{*}{$\begin{array}{l}\text { Husband's } \\
\text { education }\end{array}$} & No education & $\mathrm{I}, 246(38.12)$ \\
\hline & Primary & I,306 (39.97) \\
\hline & Secondary & 382 (II.68) \\
\hline & Higher & $311(9.52)$ \\
\hline & Do not know & $23(0.7 \mathrm{I})$ \\
\hline \multirow{12}{*}{$\begin{array}{l}\text { Administrative } \\
\text { region }\end{array}$} & Tigray & $265(7.54)$ \\
\hline & Afar & $12(0.33)$ \\
\hline & Amhara & I, I 37 (32.39) \\
\hline & Oromia & I,057 (30.10) \\
\hline & Somali & $4(0.11)$ \\
\hline & Benishangul & $32(0.92)$ \\
\hline & Gumuz & \\
\hline & SNNPR & $793(22.60)$ \\
\hline & Gambela & $10(0.28)$ \\
\hline & Harari & $6(0.18)$ \\
\hline & Addis Ababa & $180(5.13)$ \\
\hline & Dire Dawa & $15(0.42)$ \\
\hline
\end{tabular}

(Continued)
Table 2 (Continued).

\begin{tabular}{|l|l|l|}
\hline & & Weighted Frequency (\%) \\
\hline Wealth & Poorest & $396(11.28)$ \\
& Poorer & $638(18.16)$ \\
& Middle & $730(20.79)$ \\
& Richer & $775(22.09)$ \\
& Richest & $972(27.68)$ \\
\hline
\end{tabular}

Table 3 Communication Characteristics of Reproductive Age Women Currently Using Selected Contraceptive Methods in Ethiopia, EDHS 2016

\begin{tabular}{|c|c|c|}
\hline & Categories & $\begin{array}{l}\text { Weighted } \\
\text { Frequency (\%) }\end{array}$ \\
\hline $\begin{array}{l}\text { Frequency of reading } \\
\text { newspaper }\end{array}$ & $\begin{array}{l}\text { Not at all } \\
\text { Less than once a week } \\
\text { At least once a week }\end{array}$ & $\begin{array}{l}3,115(88.7 \mathrm{I}) \\
297(8.46) \\
99(2.83)\end{array}$ \\
\hline $\begin{array}{l}\text { Frequency of listening to } \\
\text { radio }\end{array}$ & $\begin{array}{l}\text { Not at all } \\
\text { Less than once a week } \\
\text { At least once a week }\end{array}$ & $\begin{array}{l}2,309(65.78) \\
575(16.39) \\
626(17.83)\end{array}$ \\
\hline $\begin{array}{l}\text { Frequency of watching } \\
\text { television }\end{array}$ & $\begin{array}{l}\text { Not at all } \\
\text { Less than once a week } \\
\text { At least once a week }\end{array}$ & $\begin{array}{l}2,502(71.27) \\
460(13.09) \\
549(15.64)\end{array}$ \\
\hline Own a mobile telephone & $\begin{array}{l}\text { No } \\
\text { Yes }\end{array}$ & $\begin{array}{l}2,549(72.59) \\
962(27.4 I)\end{array}$ \\
\hline Use of Internet & $\begin{array}{l}\text { Never } \\
\text { Yes }\end{array}$ & $\begin{array}{l}3,403(96.92) \\
108(3.08)\end{array}$ \\
\hline $\begin{array}{l}\text { Heard about family planning } \\
\text { on radio in last few months }\end{array}$ & $\begin{array}{l}\text { No } \\
\text { Yes }\end{array}$ & $\begin{array}{l}2,569(73.18) \\
942(26.82)\end{array}$ \\
\hline $\begin{array}{l}\text { Heard about family planning } \\
\text { on television in last few } \\
\text { months }\end{array}$ & $\begin{array}{l}\text { No } \\
\text { Yes }\end{array}$ & $\begin{array}{l}2,875(81.88) \\
636(18.12)\end{array}$ \\
\hline $\begin{array}{l}\text { Read about family planning } \\
\text { in newspaper in last few } \\
\text { months }\end{array}$ & $\begin{array}{l}\text { No } \\
\text { Yes }\end{array}$ & $\begin{array}{l}3,347(95.34) \\
164(4.66)\end{array}$ \\
\hline $\begin{array}{l}\text { Read about family planning } \\
\text { on text message on mobile } \\
\text { phone }\end{array}$ & $\begin{array}{l}\text { No } \\
\text { Yes }\end{array}$ & $\begin{array}{l}3,430(97.70) \\
81(2.30)\end{array}$ \\
\hline $\begin{array}{l}\text { Read about family planning } \\
\text { in a pamphlet/poster/leaflet }\end{array}$ & $\begin{array}{l}\text { No } \\
\text { Yes }\end{array}$ & $\begin{array}{l}3,320(94.57) \\
191(5.43)\end{array}$ \\
\hline $\begin{array}{l}\text { Heard about family planning } \\
\text { at community event/ } \\
\text { conversation }\end{array}$ & $\begin{array}{l}\text { No } \\
\text { Yes }\end{array}$ & $\begin{array}{l}1,948(55.47) \\
1,563(44.53)\end{array}$ \\
\hline $\begin{array}{l}\text { Seen anything about family } \\
\text { planning on the Internet }\end{array}$ & $\begin{array}{l}\text { No } \\
\text { Yes }\end{array}$ & $\begin{array}{l}3,471(98.86) \\
40(1.14)\end{array}$ \\
\hline
\end{tabular}


informed choice of contraceptives was $36.2 \%(95 \% \mathrm{CI}$ $34.6 \%-37.8 \%)$.

Multivariate analysis showed that age, religion, husband's occupation, region, frequency of watching television, source of contraceptive method, and visiting health facilities in the 12 months prior to the survey were associated with informed choice. With each year increase in age, the odds of informed choice increased 1.02 times (95\% CI 1.01-1.03). Women affiliated with other religions were less likely to be informed than those affiliated with orthodox religion (AOR 0.50, 95\% CI 0.26-0.98). Compared to those with no education, those with primary education were $24 \%$ less likely informed of methods (AOR 0.76, 95\% CI 0.62-0.93). Even though it was not significant, women with higher education had 1.57 times the odds of informed choice of those with no education (AOR 1.57, 95\% CI 0.96-2.57). Women whose husbands worked in agriculture (AOR $0.66,95 \%$ CI $0.46-0.94$ ), unskilled jobs (AOR 0.46, 95\% CI 0.26-0.79), or did not know their husband's job (AOR 0.45, 95\% CI 0.26-0.79) had lower odds of informed choice of methods than those with an unemployed husband. Women living in the Amhara (AOR 0.35, 95\% CI 0.25-0.49) and Oromia (AOR $0.68,95 \%$ CI 0.48-0.97) regions had lower odds of informed choice than Tigray region residents. Women who watched television less than once a week (AOR 1.37, 95\% CI 1.05-1.79) had 1.37 times the odds of informed choice of those who did not watch television. Nevertheless, women who watched television at least once a week the $33 \%$ lower odds of informed choice (AOR 0.67, 95\% CI 0.47-0.97) of those who did not watch television. Women receiving contraceptive methods from private clinics (AOR 0.48, 95\% CI 0.37-0.62) and pharmacies (AOR 0.50, 95\% CI 1.90-24.81) had lower odds of informed choice than those receiving them from government institutions. Women visiting health facilities in the last 12 months prior to the study (AOR 1.47, 95\% CI 1.25-1.73) had 1.47 times the odds of informed choice as their counterparts (Table 4).

\section{Discussion}

This study examined the level of informed choice of contraceptive methods among reproductive-age women in Ethiopia. Given previous studies, this study has indirectly highlighted experiences of health-care providers working in FP on providing information on methods. ${ }^{18}$ Women should feel involved and informed, so that they can make informed choices about their care. ${ }^{19}$ To support people's right to make an informed choice about contraceptive methods, FP programs must offer clients ready access to a range of methods, complete, and accurate information about these methods, and help in weighing the options. ${ }^{11}$

According to this study, $36.2 \%$ of the women made an informed choice of contraceptive methods. This finding highlights that a significant number of women using contraception were uninformed about side effects and other methods available. This lack of deeper knowledge needed to make an informed choice may result in discontinuation of service use. ${ }^{20}$ This might be due to inadequate communication and poor client-provider interaction. ${ }^{20,21}$ Improving contraceptive counseling and ensuring supplies for day-to-day service delivery can improve the quality of care that clients receive and enable women to make an informed choice.

In this study, below half (45.5\%) the participants talked about side effects of the methods, and only $32.5 \%$ knew how to manage side effects if experienced. Similarly, $55.6 \%$ were informed about other potential methods. This finding is lower than a study done in rural India. ${ }^{18}$ This might be due to differences in health-service availability, and socioeconomic and cultural factors. In addition to the difference in study setting, the study conducted in India was facility-based. These findings suggest that the government needs to improve the extent of informed choice of methods by working on the quality of FP counseling and provider-client communication.

Decisions women made on FP methods reflect a variety of influences. ${ }^{22}$ In this study, several factors were identified that affected choice of contraceptive methods among reproductive-age women in Ethiopia. Increased age, watching television less than once a week, and visiting health facilities in the last 12 months contributed to increased informed choice. With regard to age, a study done elsewhere highlighted that higher informed choice was observed among older age-groups than their younger counterparts. This may be due to cumulative learning as age increases. In addition, women who visited health facilities in the last 12 months period may have had current information and were able to remember the conversations they had with health workers, which increased informed choice.

Being affiliated with the Orthodox religion, primary education, living in Amhara or Oromia region, watching television at least once a week, and receiving contraceptives from private clinics and pharmacies were associated with lower informed choice of contraceptive methods. 
Table 4 Factors Associated with Informed Choice of Contraceptive Among Reproductive Age Women Currently Using Selected Contraceptive Methods in Ethiopia, EDHS 2016

\begin{tabular}{|c|c|c|c|}
\hline \multicolumn{2}{|l|}{ Explanatory Variable } & \multicolumn{2}{|c|}{ Informed Choice of Contraceptive Methods } \\
\hline & & Crude OR $(95 \% \mathrm{Cl})$ & Adjusted OR $(95 \% \mathrm{Cl})$ \\
\hline Age $^{* *}$ & & $1.02(1.01,1.03)$ & $1.02(1.01,1.03)^{*}$ \\
\hline Residence & $\begin{array}{l}\text { Urban } \\
\text { Rural }\end{array}$ & $\begin{array}{l}\text { I } \\
0.56(0.48,0.66)\end{array}$ & $\begin{array}{l}1 \\
0.94(0.68,1.29)\end{array}$ \\
\hline Religion & $\begin{array}{l}\text { Orthodox } \\
\text { Muslim } \\
\text { Protestant } \\
\text { Other }\end{array}$ & $\begin{array}{l}1 \\
0.86(0.72,1.03) \\
1.18(1.00,1.39) \\
0.60(0.33,1.12)\end{array}$ & $\begin{array}{l}\text { I } \\
0.83(0.66,1.04) \\
0.96(0.74,1.24) \\
0.50(0.26,0.98)^{*}\end{array}$ \\
\hline Education & $\begin{array}{l}\text { None } \\
\text { Primary } \\
\text { Secondary } \\
\text { Higher }\end{array}$ & $\begin{array}{l}1 \\
0.93(0.80,1.09) \\
1.52(1.20,1.93) \\
2.54(1.92,3.36)\end{array}$ & $\begin{array}{l}\text { I } \\
0.76(0.62,0.93)^{*} \\
1.05(0.74,1.50) \\
1.57(0.96,2.57)\end{array}$ \\
\hline Occupation & $\begin{array}{l}\text { Not working } \\
\text { Professional } \\
\text { Agriculture } \\
\text { Unskilled } \\
\text { Others }\end{array}$ & $\begin{array}{l}\text { I } \\
1.37(1.16,1.61) \\
0.73(0.61,0.88) \\
1.14(0.60,2.17) \\
1.47(0.93,2.32)\end{array}$ & $\begin{array}{l}\text { I } \\
0.93(0.70,1.24) \\
0.89(0.69,1.14) \\
\text { I.08 }(0.49,2.38) \\
1.19(0.66,2.17)\end{array}$ \\
\hline Husband's education & $\begin{array}{l}\text { None } \\
\text { Primary } \\
\text { Secondary } \\
\text { Higher } \\
\text { Do not know }\end{array}$ & $\begin{array}{l}\text { I } \\
1.42(1.20,1.67) \\
1.70(1.34,2.15) \\
2.51(1.95,3.23) \\
1.25(0.53,2.97)\end{array}$ & $\begin{array}{l}\text { I } \\
\text { I.20(0.99, I.46) } \\
\text { I.18(0.86, I.6I) } \\
\text { I.28(0.86, I.90) } \\
\text { I.38(0.54, 3.54) }\end{array}$ \\
\hline Husband's occupation & $\begin{array}{l}\text { Not working } \\
\text { Professional } \\
\text { Agriculture } \\
\text { Unskilled } \\
\text { Others }\end{array}$ & $\begin{array}{l}\text { I } \\
\text { I.I7 }(0.83,1.65) \\
0.60(0.43,0.84) \\
0.69(0.42,1.15) \\
0.78(0.48,1.26)\end{array}$ & $\begin{array}{l}\text { I } \\
0.82(0.55,1.20) \\
0.66(0.46,0.94)^{*} \\
0.46(0.26,0.79)^{*} \\
0.45(0.26,0.79)^{*}\end{array}$ \\
\hline Administrative region & $\begin{array}{l}\text { Tigray } \\
\text { Afar } \\
\text { Amhara } \\
\text { Oromia } \\
\text { Somali } \\
\text { Benishangul Gumuz } \\
\text { SNNPR } \\
\text { Gambela } \\
\text { Harari } \\
\text { Addis Ababa } \\
\text { Dire dawa }\end{array}$ & $\begin{array}{l}\text { I } \\
0.35(0.09,1.29) \\
0.31(0.23,0.4 I) \\
0.53(0.40,0.69) \\
0.71(0.09,5.31) \\
0.52(0.25,1.11) \\
0.59(0.44,0.78) \\
0.28(0.06,1.23) \\
1.35(0.27,6.77) \\
1.25(0.86,1.84) \\
1.27(0.44,3.67)\end{array}$ & $\begin{array}{l}\text { I } \\
0.38(0.09,1.72) \\
0.35(0.25,0.49)^{*} \\
0.68(0.48,0.97)^{*} \\
1.26(0.14,11.26) \\
0.75(0.33,1.73) \\
0.68(0.46,1.00) \\
0.39(0.08,2.04) \\
1.20(0.20,7.31) \\
1.27(0.78,2.07) \\
1.28(0.38,4.34)\end{array}$ \\
\hline Wealth & $\begin{array}{l}\text { Poorest } \\
\text { Poorer } \\
\text { Middle } \\
\text { Richer } \\
\text { Richest }\end{array}$ & $\begin{array}{l}\text { I } \\
0.71(0.54,0.93) \\
0.86(0.66,1.11) \\
1.20(0.93,1.54) \\
1.71(1.34,2.18)\end{array}$ & $\begin{array}{l}\text { I } \\
0.77(0.57,1.04) \\
0.93(0.70,1.24) \\
\text { I.23( }(0.92,1.65) \\
\text { I.40(0.98, } 2.01)\end{array}$ \\
\hline Frequency of reading newspaper & $\begin{array}{l}\text { Not at all } \\
\text { Less-than once a week } \\
\text { At least once a week }\end{array}$ & $\begin{array}{l}\text { I } \\
2.01(1.59,2.56) \\
2.27(1.52,3.39)\end{array}$ & $\begin{array}{l}\text { I } \\
1.04(0.75,1.45) \\
0.99(0.60,1.66)\end{array}$ \\
\hline
\end{tabular}

(Continued) 
Table 4 (Continued).

\begin{tabular}{|c|c|c|c|}
\hline \multicolumn{2}{|l|}{ Explanatory Variable } & \multicolumn{2}{|c|}{ Informed Choice of Contraceptive Methods } \\
\hline & & Crude OR $(95 \% \mathrm{Cl})$ & Adjusted OR $(95 \% \mathrm{Cl})$ \\
\hline Frequency of listening to radio & $\begin{array}{l}\text { Not at all } \\
\text { Less than once a week } \\
\text { At least once a week }\end{array}$ & $\begin{array}{l}1 \\
1.39(1.15,1.68) \\
1.85(1.54,2.21)\end{array}$ & $\begin{array}{l}1 \\
0.90(0.71,1.15) \\
1.01(0.79,1.30)\end{array}$ \\
\hline Frequency of watching television & $\begin{array}{l}\text { Not at all } \\
\text { Less than once a week } \\
\text { At least once a week }\end{array}$ & $\begin{array}{l}\text { I } \\
1.56(1.27,1.91) \\
1.69(1.40,2.04)\end{array}$ & $\begin{array}{l}\text { I } \\
\text { I.37 (I.05, I.79)* } \\
0.67(0.47,0.97)^{*}\end{array}$ \\
\hline Source of FP method & $\begin{array}{l}\text { Government institutions } \\
\text { Private clinic/delivery } \\
\text { Pharmacy } \\
\text { NGO } \\
\text { Others }\end{array}$ & $\begin{array}{l}\text { I } \\
0.55(0.44,0.70) \\
0.68(0.43,1.07) \\
1.96(1.07,3.59) \\
0.06(0.01,0.66)\end{array}$ & $\begin{array}{l}\text { I } \\
0.48(0.37,0.62)^{*} \\
0.50(0.29,0.87)^{*} \\
1.02(0.50,2.08) \\
0.07(0.01,1.03)\end{array}$ \\
\hline Heard about FP on radio in last few months & $\begin{array}{l}\text { No } \\
\text { Yes }\end{array}$ & $1.98(1.70,2.30)$ & $\begin{array}{l}\text { I } \\
1.25(1.00,1.55)\end{array}$ \\
\hline Heard about FP on television in last few months & $\begin{array}{l}\text { No } \\
\text { Yes }\end{array}$ & $\begin{array}{l}1 \\
1.98(1.66,2.35)\end{array}$ & $\begin{array}{l}1 \\
1.00(0.72,1.38)\end{array}$ \\
\hline Read about FP in newspaper in last few months & $\begin{array}{l}\text { No } \\
\text { Yes }\end{array}$ & $\begin{array}{l}\text { I } \\
2.74(1.99,3.78)\end{array}$ & $\begin{array}{l}\text { I } \\
\text { I.47 }(0.95,2.26)\end{array}$ \\
\hline Read about FP on text message on mobile phone & $\begin{array}{l}\text { No } \\
\text { Yes }\end{array}$ & $\begin{array}{l}\text { I } \\
2.20(1.39,3.38)\end{array}$ & $\begin{array}{l}\text { I } \\
1.03(0.61,1.76)\end{array}$ \\
\hline Read about FP in pamphlet/poster/leaflet & $\begin{array}{l}\text { No } \\
\text { Yes }\end{array}$ & $2.54(1.89,3.42)$ & I. $10(0.74,1.65)$ \\
\hline Heard about FP at community event/conversation & $\begin{array}{l}\text { No } \\
\text { Yes }\end{array}$ & $1.53(1.34,1.76)$ & I. $1.49(1.26,1.75)$ \\
\hline Seen anything about FP on the Internet? & $\begin{array}{l}\text { No } \\
\text { Yes }\end{array}$ & $3.13(1.64,5.99)$ & I. \\
\hline Respondent currently working & $\begin{array}{l}\text { No } \\
\text { Yes }\end{array}$ & $\begin{array}{l}\mathrm{I} \\
1.67(1.45,1.92)\end{array}$ & $\begin{array}{l}\mathrm{I} \\
\mathrm{I} .01(0.78,1.31)\end{array}$ \\
\hline Visited health facility in last 12 months & $\begin{array}{l}\text { No } \\
\text { Yes }\end{array}$ & $\begin{array}{l}\text { I } \\
1.41(1.22,1.62)\end{array}$ & $\begin{array}{l}\text { I } \\
1.47(1.25,1.73)^{*}\end{array}$ \\
\hline
\end{tabular}

Notes: $* P<0.05 ; * *$ continuous variable.

Unexpectedly, primary education and watching television at least once a week were associated with lower informed choice. Even though it was not significant, women with higher education had higher odds of informed choice. This might be because some women use pharmacies only and overlook the counseling sessions. Media exposure for women may be affected by media preference. In contrast, this might be due to other confounding factors. However, previous studies have revealed the availability of information in the media, access to FP services, cultural, religious, and social influences, socioeconomic status, and community standards affects informed choice of contraceptives. ${ }^{22}$
Since these two regions (Oromia and Amhara) are the most populated in the nation, the regional variation may be attributed to access to health facilities and professional: client ratio, but this needs further study. Conversely, cultural and religious factors may contribute to variation in informed choice. Evidence has revealed that the husband plays an important role in women's health and contraceptive use. ${ }^{24}$ In this study, having a husband working in agricultural, unskilled jobs and not knowing the husband's job demonstrated lower informed choice. Commonly, uneducated people engaged in such jobs (agriculture and unskilled) may linked with reduced focus on women's 
health and health decisions, which may lead to lower informed choice. This may highlight lower involvement of men in $\mathrm{FP}^{24}$

Strengthening FP counseling, technical knowledge, and interpersonal communication skills alone are not sufficient to address the gaps in informed choice of contraceptive methods. ${ }^{11}$ In many settings, informed choice is constrained by lack of understanding of the concept of informed choice, as well as by social norms that limit women's and adolescents' ability to assert their rights. ${ }^{11,12,23}$ On the other hand, women may not understand the options, have trouble in weighing the pros and cons, and may not identify the most important attributes of the methods. In such cases, women often find it difficult to make a decision and need support in the decision-making process. FP-service providers and programmers need to find a balance between the client's and the provider's input into the decision-making process. ${ }^{25}$ Achieving these calls for a mix of interventions directed at both providers and clients. ${ }^{11}$

\section{Strengths and Limitations}

The strength of this study was that it used national representative data. Regarding limitations, as with any crosssectional study, causality was not possible to establish for the factors covered. The other limitation is recall bias, which might occur due to memory lapse. Moreover, the finding on informed choice represents only the contraceptive methods selected (female sterilization, implants, injections, IUD, and the pill).

\section{Conclusion}

Informed choice for contraceptives was delayed back to the increased modern contraceptive use. A considerable proportion of reproductive-age women in Ethiopia were inadequately informed about side effects and/or other methods available. Only $36.2 \%$ of women made an informed choice of contraceptive method. Age, religion, husband's occupation, administrative region, frequency of watching television, source of FP, and visiting a health facility in the last 12 months were factors significantly associated with informed choice. Therefore, health-care facilities should focus on enabling women to make an informed choice of contraception method to secure the continual use of the service. Providers also ought to use counseling sessions to help women make a thoughtful decision on contraceptives. Further studies also need to be carried out on quality FP-counseling services. Private health facilities also need to give emphasis to delivering essential messages about methods that provide and assure women's rights and choices. On the other hand, community-based awareness activities need to be strengthened.

\section{Abbreviations}

DHS, Demographic and Heath Surveys; EDHS, Ethiopia Demographic and Heath Surveys; FP, family planning; IUD, intrauterine contraceptive device; SNNPR, Southern Nations, Nationalities, and Peoples' Region; WHO, World Health Organization.

\section{Data Sharing Statement}

The raw data used in this study can be accessed from the DHS website: https://dhsprogram.com/data/availabledatasets.cfm.

\section{Ethics Approval and Consent to Participate}

Data were accessed from the DHS website (http://www. measuredhs.com) after registration and permission was obtained. As published in the EDHS report of 2016, verbal informed consent was obtained from all participants ${ }^{16}$ and all identifiers removed.

\section{Acknowledgments}

The author would like to acknowledge the Measure DHS data archive and International Classification of Functioning, Disability, and Health International for permission to use the data.

\section{Disclosure}

The author declares that there are no conflicts of interest.

\section{References}

1. UNFPA. Programme of Action of the International Conference on Population Development, 20th Anniversary Edition; 2014

2. Rimon JG II, Tsui AO. Regaining momentum in family planning. Glob Health Sci Pract. 2018;6(4):626-628. doi:10.9745/GHSP-D-18-00483

3. Cleland J, Harbison S, Shah IH. Unmet need for contraception: issues and challenges. Stud Fam Plann. 2014;45(2):105-122. doi:10.1111/ j.1728-4465.2014.00380.x

4. The RESPOND Project: a fine balance: contraceptive choice in the 21st century-an action agenda. Report of the September 2012 Bellagio conference. New York: Engender Health/The RESPOND Project; 2013.

5. Kim YM, Kols A, Mucheke S. Informed choice and decision-making in family planning counseling in Kenya. Int Fam Plan Perspect. 1998;1:2-4. doi:10.2307/2991913 
6. World Health Organization. Ensuring human rights within contraceptive programmes: a human rights analysis of existing quantitative indicators; 2014.

7. World Health Organization. Ensuring human rights in the provision of contraceptive information and services: guidance and recommendations; 2014.

8. Stanback J, Steiner M, Dorflinger L, Solo J, Cates W. WHO tiered-effectiveness counseling is rights-based family planning. Glob Health Sci Pract. 2015;3(3):352-357.

9. UN Committee on Economic, Social and Cultural Rights. UN Doc. E/C.12/2000/4. General Comment No. 14, The right to the highest attainable standard of health; 2000

10. Loaiza E, Liang M, Snow R. Informed choice for modern contraceptive use: evidence from 24 countries served by the UNFPA supplies programme. Population Association of America meeting; March 2016.

11. PATH and United Nations Population Fund. Meeting the Need: Strengthening Family Planning Programs. Seattle: PATH/UNFPA; 2006.

12. Engender Health. Choices in family planning: informed and voluntary decision making. New York: Engender Health; 2003b. Available from: www.engenderhealth.org/res/offc/ic/choices/. Accessed February 23, 2021.

13. Fruhauf T, Zimmerman L, Kibira SP, et al. Measuring family planning quality and its link with contraceptive use in public facilities in Burkina Faso, Ethiopia, Kenya and Uganda. Health Policy Plan. 2018;33(7):828-839. doi:10.1093/heapol/czy058

14. DKT Ethiopia. Ethiopia family planning landscape. Available from: https://www.dktethiopia.org/sites/default/files/PublicationFiles/ Ethiopia\%20Landscape\%20v9.2.pdf. Accessed February 23, 2021.

15. Tafese F, Woldie M, Megerssa B. Quality of family planning services in primary health centers of Jimma Zone, Southwest Ethiopia. Ethiop J Health Sci. 2013;23(3):245-254. doi:10.4314/ejhs.v23i3.7

16. Central Statistical Agency (CSA) [Ethiopia] and ICF. Ethiopia Demographic and Health Survey 2016. Addis Ababa, Ethiopia, and Rockville, Maryland, USA: CSA and ICF; 2016.
17. Castle S, Askew I. Contraceptive discontinuation: reasons, challenges, and solutions; 2015.

18. Mozumdar A, Gautam V, Gautam A, et al. Choice of contraceptive methods in public and private facilities in rural India. BMC Health Serv Res. 2019;19(1):421. doi:10.1186/s12913-019-4249-0

19. World Health Organization Department of Reproductive Health and Research (WHO/RHR) and Johns Hopkins Bloomberg School of Public Health/Center for Communication Programs (CCP), Knowledge for Health Project. Family Planning: A Global Handbook for Providers (2018 Update). Baltimore and Geneva: CCP and WHO; 2018.

20. Dansereau E, Schaefer A, Hernández B, et al. Perceptions of and barriers to family planning services in the poorest regions of Chiapas, Mexico: a qualitative study of men, women, and adolescents. Reprod Health. 2017;14(1):129. doi:10.1186/s12978-017-0392-4

21. Nelumbu PN, Amakali K, Hofnie-Hoëbes K. Application of elements of the informed choice of modern contraceptives among reproductive aged women in the Khomas region of Namibia. J Nursing Educ Practice. 2015;6. doi:10.5430/jnep.v6n2p130

22. Valdés PR, Alarcon AM, Munoz SR. Evaluation of Informed Choice for contraceptive methods among women attending a family planning program: conceptual development; a case study in Chile. J Clin Epidemiol. 2013;66(3):302-307. doi:10.1016/j.jclinepi.2012.07.003

23. Murphy E, Steele C. Client-provider interactions in family planning services: guidance from research and program experience. Maximizing Access and Quality Paper. 2000. 1(2)

24. Chekole MK, Kahsay ZH, Medhanyie AA, Gebreslassie MA, Bezabh AM. Husbands' involvement in family planning use and its associated factors in pastoralist communities of Afar, Ethiopia. Reprod Health. 2019;16(1):1-7. doi:10.1186/s12978-019-0697-6

25. Kim YM, Kols A, Martin A, et al. Promoting informed choice: evaluating a decision-making tool for family planning clients and providers in Mexico. Int Fam Plan Perspect. 2005;31(4):162-171. doi: $10.1363 / 3116205$
Open Access Journal of Contraception

\section{Publish your work in this journal}

Open Access Journal of Contraception is an international, peerreviewed, open access, online journal, publishing original research, reports, reviews and commentaries on all areas of contraception. In addition to clinical research, demographics and health-related aspects, the journal welcomes new findings in animal and preclinical

\section{Dovepress}

studies relating to understanding the biological mechanisms and practical development of new contraceptive agents. The manuscript management system is completely online and includes a very quick and fair peer-review system. Visit http://www.dovepress.com/testimonials. php to read real quotes from published authors. 